\title{
Workplace Health Promotion: Effects of an mHealth application on Employee Behaviour and Wellness
}

\author{
Sophie Emerson \\ University College Cork \\ sophieemerson@gmail.com
}

\author{
Ciara Heavin \\ University College Cork \\ c.heavin@ucc.ie
}

\author{
Daniel J. Power \\ University of Northern Iowa \\ daniel.power@uni.edu
}

\begin{abstract}
Organizations are using digital tools to support workplace health promotion (WHP) initiatives. This study investigates the effects of a mobile health (mHealth) application on employee behaviour and wellness. This exploratory, empirical case study was conducted as part of an established workplace health initiative in the Health Service Executive (HSE) in Ireland, April 2018. User data on exercise including daily activity in the form of steps taken, mood, total daily exercise, and other variables were captured as part of the 'Steps to Health Challenge'. Phase 1 of the study involved the collection of user data from 70 active app users and a survey was conducted. The majority of participants found that the mHealth app motivated them to sustain, and in some cases increase, their daily steps. Phase 2 involved interviews. Following qualitative data analysis, we found that the app positively effected employee behaviour; specific features were highlighted as especially influential.
\end{abstract}

\section{Introduction}

The World Health Organisation (WHO) is supporting workplace health promotion initiatives [1]. In 2010, the WHO proposed a model of a healthy workplace continual improvement process. Large companies such as Google, Microsoft, and Accenture seem to recognize the potential benefits of workplace initiatives to promote health, happiness, and productivity and run large-scale employee WHP activities [2].

Studies have shown that workplace health initiatives can reduce absenteeism and medical costs [3]. These initiatives include healthy eating promotions [4], encouragement of active lifestyles [5], and awareness of mental health issues [6]. The Irish Heart foundation promote a number of workplace health initiatives. Its "Healthy Eating Awards" encourage workplaces to offer nutritious food options and their "Active@work" programme supports and motivates employees to get more active [5].

Health initiatives can benefit an individual as well as a company [3]. Evidence suggests that an increase in employee activity can positively effect an individual's mental wellbeing [6] as well as one's physical health [4]. A "Steps Challenge" is a popular corporate health initiative [7] that also serves to benefit individuals. Many employees work in a naturally sedentary setting. Sitting for long periods, as many office workers do, has negative consequences on one's health [8].

Initiating a workplace health and wellness initiative does not guarantee employee participation or improved overall health of an organization's workforce. More research needs to investigate what works when and under what conditions, especially in the area of mobile health (mHealth) data collection and feedback. The purpose of this exploratory study was to investigate the effect of an innovative mHealth activity tracking platform provided as part of a public sector, workplace health initiative. Specifically, this research investigates gaps in our understanding of the impacts of using an mHealth app in a structured workplace health program, on the internal motivation to improve one's health and how external motivation impacts employee physical activity levels and attitudes. Section 2 presents a review of the activity-based tracking technology literature and Section 3 considers examples of these within the context of WHP initiatives. Section 4 outlines the research method. The results of the study are presented in Section 5. Section 6 discusses the results and limitations of the study. Finally, the conclusions are outlined in Section 7. Overall, this study demonstrates that more design science research is needed in the area of mHealth.

\section{2. mHealth Activity-Based Tracking Technologies}

Improvements in sensor-based technologies have positively impacted the use of activity-based tracking 
devices among individuals [9]. These technologies may be a distinct data capture device e.g. FITBIT or Jawbone paired with an app or it might be a smart phone paired with one of the many available tracking software applications [9]. Smart phones are becoming a reliable way of tracking fitness data as built in accelerometers, gyroscopes, and GPS are standard features [10]. Activity-based tracking technology has enabled the collection, storage, and analysis of individual health and wellbeing data in real-time [10]. The main objective of these technologies is to effect positive behavioural change in people, for example increase exercising, improve habits such as eating, spending, and studying that will improve desired outcomes $[9,11,12]$.

Activity-based tracking technologies contribute to the phenomenon referred to as the "quantified self" [9]. Quantified Self refers to any kind of biological, physical, behavioral, or environmental information self-tracking either as an individual or in a group, with the objective of improving self-sensing, self-awareness, and human performance [13]. More recently, the term "Personal Informatics" has also been used to describe the collection, storage, analysis, and visualization of sensor collected data [9]. This includes a variety of data such as financial, video or social media diaries, exercise, health, and nutrition that may be collected through a diverse range of apps and sensors [9]. Specifically, James and colleagues define fitness technology "as any combination of hardware and software that enables the collection, storage, analysis, socialization, and management of individuals' health and wellness data, including but not limited to exercise activity, nutrition, and sleep data" [9:1011]. Studies have shown that maintaining engagement with health/fitness based tracking technology is difficult [14]. Recent research indicates that people lapse in their use of tracking tools over time [14]. Abandonment may be attributed to poor design, unreliability, diminished interest in exercise or selftracking, or loss of the device [15]. Knowing which technology features are most effective and understanding the factors that motivate the user to maintain their engagement is important. The design of activity-based tracking technology is integral to the success of the technology [9, 14]. Design includes the visual aesthetics of the software and hardware, as well as the perceived accuracy or performance of the technology [15].

Users intrinsic and extrinsic exercise goals are associated with the features of the technology being used [9]. According to [16] it is important to distinguish between the intrinsic and extrinsic motives of individuals and to determine how different stimuli help fulfil them. An individual's personal or intrinsic motivation to engage with the technology is important [17]. Intrinsic motivation might include factors such as joy, self-efficacy, and control [17]. In terms of maintaining engagement with health related activitybased tracking, this might include supporting an individual user to set their own goals and motivate them to achieve these through tailored messages and rewards such as badges, levels, and progress bars [18]. The social motivation or extrinsic factors [17] to maintain the use of an activity tracking technology is also a key factor. This is important, particularly in terms of leveraging the affordances of activity-based tracking technology features to promote social comparison. Social comparison can act as a prompt to motivate positive behaviour change in a group setting [14]. Features of an activity-based tracking tool can support social engagement with others encouraging people to stay active $[14,19]$. These features include the ability to share your personal progress with a friend [19], group tracking via performance charting [14], and group messaging [14]. Social comparison through the use of these group-based features promotes both competition and socialization among users [9, 14, 19]. While this is a growing research area, limited empirical studies have been undertaken exploring the use of mHealth activity-based tracking technologies in workplace health promotion (WHP).

\section{Examples of Workplace Health Initiatives and Activity-Based Tracking}

Research shows that WHP initiatives, which combat sedentary behaviour, can have a positive impact on a participant's BMI, waist girth and resting heart rate [20]. There are many reasons for WHP initiatives. The obvious one is the possible health benefits for employees that participate in these programs. Workplace health initiatives can take different forms including one-on-one interventions, group sessions, they can be activity-based and mental health focused. Workplace health promotions tend to focus on a single theme such as increased activity [3], a particular illness or on changing personal health practices and behaviours e.g. smoking, diet [7]. Some studies indicate that tailored or individualized WHP initiatives have proven to be more successful than semi-tailored or general programs [21], however these initiatives can be resource intensive.

While WHP initiatives are not new [7], utilising mHealth technology, i.e. activity-based tracking technology, as a tool to capture and analyse employee health and wellbeing data is a relatively recent initiative [10]. A study by a team at Portland Oregon Health and Science University, sought to "evaluate the 
effects of a research-tested, team-based health promotion and wellness program combined with digital technologies and implemented in a diverse worksite setting among hospital, clinic, and university employees" [7:1]. This wellness program positively effected participant's blood pressure, body, weight, BMI, diet, mood, stress levels, and sleep quality [7].

Limited research has been carried out in this area, with the majority of studies focusing on the physical health aspect of these initiatives [22]. mHealth technologies used in conjunction with smart phone technology has the potential to sustain a meaningful positive impact on an individual's health [22]. In one study, participants indicated that integration with their smart phone, ease of application use and feedback on activity progress could sustain a positive change in lifestyle [22]. Participants in this study found "simplicity, integration into daily life, and clear feedback on progress" [22] key features in personal health technologies.

Results from a pedometer-based community walking intervention in Scotland, saw increases in time spent walking for leisure and a decrease in time spent sitting [8]. This study had 79 participants record their steps for 12 weeks using a traditional pedometer. Similar to this was the 2013 "Walk@work" scheme, undertaken by nearly 400 staff and students from Queensland University. The study concluded that after the six-week program, walking at work had increased by approximately $25 \%$ [23].

One aspect of a person's wellbeing is their physical health, but happiness and mental state also play an important role [6]. Research on digital health interventions to support mental health is not as common, but studies have indicated that exercise correlates positively with an elevation in the psychological wellbeing of an individual [7]. Existing research indicates that some electronic health interventions can be effective at reducing mental health and stress symptoms [7].

In a 2017 US study, 281 university staff members took part in a fitness program consisting of three cardiovascular conditioning and strength training workouts per week for 12 weeks, run by fitness trainers in groups of 20-40 year olds [6]. The results revealed significant improvements across physical and mental health, stress, energy level, social satisfaction, selfefficacy, and quality of life [6].

While mHealth technologies show promise in providing people with low-cost, immediate support for their mental health [24], there is little empirical data to help us understand the use of activity-based tracking technology as part of a targeted employee health programme. The focus of this study is to provide more detailed information about what motivates individual employees to use the technology, what technology features are used and when, and how the features impact the social engagement among a group of employees as part of a workplace health promotion initiative. We hypothesized that the mHealth application would have a positive effect on participants and that participants would perceive that they would be better able to track their daily activity using the app.

\section{Methodology}

This exploratory empirical single case study was conducted as part of an established workplace health initiative in the Health Service Executive (HSE) in Ireland. The HSE provides public health and social care services to everyone living in Ireland. This research focuses on employee behaviour and wellness in a homogenous population, namely public health service employees.

\subsection{Setting, study population and participant recruitment}

This research emerged from a collaboration between the Health \& Wellbeing, Human Resources and Communications divisions within the HSE. In April/May 2016 the HSE Healthy Eating Active Living Programme ran a 5-week "Steps to Health Challenge", inviting all staff (circa 116,000) across 2,500 worksites to participate; with a target of $1 \%$ employee participation. Actual participation was in excess of 5,000 participants. This challenge encouraged HSE employees to use a pedometer to track their daily steps and record.

In 2018, approximately 6,500 employees took part in the challenge. Of these participants, one hundred volunteers were recruited from three divisions (Health \& Wellbeing, Human Resources, and Communications) to try an mHealth activity-based tracking software "app" to track their activity and wellness. These employees were required to register, download, and use an existing mHealth app. The app features included automatic recording of steps/activity using the phones in-built pedometer, an option to input personal data such as height/weight, a suite of automated motivational messaging about the Steps to Health Challenge, and a feature to record level of wellness (mood) at work.

We undertook a mixed method study including quantitative data collection and analysis of user activity analysis, survey, and qualitative follow up semistructured interviews with employees participating in the challenge. The integration of the quantitative and qualitative data occurred between data analysis from 
one phase and data collection from phase two [25]. There was no specific gender/age, exclusion/inclusion criteria set for participant recruitment. In Phase 1, from the 6,500 "Steps to Health Challenge Participants" a random sample of 100 participants were selected to use the mHealth app. Seventy-seven of participants activated their accounts. Seven of these accounts were missing large amounts data and were removed from the final dataset. All participants were of working age, from 20 to 65 years. Ethical approval to conduct this study was granted.

The user activity dataset was generated by challenge participants via the mHealth app. At the outset, participants were asked to input their gender, weight, height, and age. Users had the option of participating in a daily mood check-in. Mood was logged on a discrete numerical scale from one to five; this was displayed to users in the form of a series of five faces with emotions ranging from happy to sad. Users had the option to log additional exercise manually. A leaderboard feature was introduced to the mHealth app in Week 4 of the challenge.

Following the challenge based on Brooke's System Usability Scale (SUS) [26], an adapted survey (using a five point Likert scale) was distributed to capture user feedback on the mHealth app's usability and perceived usefulness in areas such as individual motivation, team building, exercise and how employees felt about work. This optional survey was completed by 42 participants through the online Survey Monkey tool. Some surveys were incomplete as questions were optional.

In Phase 2, qualitative data were collected to probe deeper with challenge participants. The interview questions were informed by survey data analysis. They were semi-structured in nature and focused on probing further on mHealth app design, the social comparison features i.e. leaderboard and mood tracking, and employees' personal attitude to using mHealth as part of a WHP. The interviews were digitally recorded, anonymised at source, transcribed, and deleted from the recording devices. Four team coordinators and two team members were interviewed; the interviews lasted approximately 131 minutes in total. Figure 1 summarizes the data gathering method.

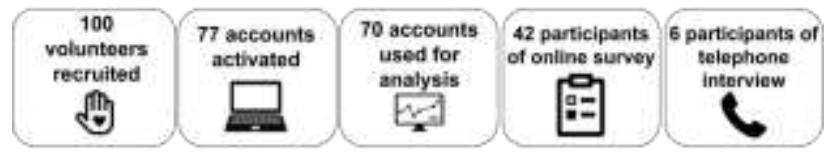

Figure 1. Summary of Data Gathering Approach

\subsection{Data Analysis}

The user-generated dataset from the mHealth app was cleaned, and exploratory analysis was carried out in MS Excel. The data was aggregated and converted into a .csv file and imported into R. Two datasets were aggregated and transported to $\mathrm{R}$. The difference between the two datasets was that one included the variable "average daily mood" and one did not. The first dataset, where the variable average daily mood was missing, consisted of 70 participants after data cleaning. The reason for creating the second dataset was the low user rate of the mood-tracking feature. This resulted in the average "daily mood variable" being vastly incomplete in terms of mood log entries. The dataset containing "average daily mood" contained the same information as the initial data set but any data with missing mood log entries were removed.

The second dataset was smaller with 56 participants. The variable "average daily steps" was compared against other variables using $\mathrm{R}$. This analysis was done to check if any notable results emerged. We checked the density distributions of the steps data, by creating histograms and density plots. We calculated the mean average daily steps of subgroups such as gender, and before/ after the leaderboard intervention was introduced to the platform. Scatter plots of variables exhibited a relationship to each other ("Average daily steps" and "Average daily mood") and the strength of this relationship was examined by calculating the correlation coefficient in $\mathrm{R}$.

Phase 1 data analysis was carried out in MS Excel by producing bar charts illustrating the survey data. As part of Phase 2, data collected from conducting six qualitative interviews were fully transcribed, proofread and open coding was conducted to identify patterns in the data [27]. In addition, we imported the text files to $\mathrm{R}$, where all non- relevant words such as "the" or "and" were removed. Word clouds and word frequency plots were produced in $\mathrm{R}$, this approach supported the qualitative analysis approach undertaken.

\section{Results}

Analysis of the data in the two phases shed light on users' perceptions about the design of the mHealth app, whether digital activity-based health tracking is perceived as being positive or negative by individual users, and to further understand its impacts on employee behaviour and wellness. The introduction of the leaderboard feature, at the beginning of week four, was examined. This unanticipated change was investigated to understand its impact on individual employee daily step count.

As Table 1 shows, most participants found that using the mHealth app helped them track their daily steps compared with the traditional paper and 
pedometer method. Of the five statements a majority of participants (over half of the 37 participants) strongly agreed/agreed with each statement. The error used in the calculations displayed in Table 1 is standard deviation (SD).

Table 1. mHealth Application survey results $(n=42)$

\begin{tabular}{|c|c|}
\hline $\begin{array}{l}\text { Statement } \\
\text { The mHealth app }\end{array}$ & $\begin{array}{l}\text { Strongly } \\
\text { Agreed/Agreed } \\
\pm \mathrm{SD} \%\end{array}$ \\
\hline $\begin{array}{l}\text {...made it easier to track their } \\
\text { activity }(n=37) \text {. }\end{array}$ & $67.5 \pm 7.7 \%$ \\
\hline $\begin{array}{l}\text {...helped them track their } \\
\text { activity accurately }(n=37) \text {. }\end{array}$ & $54.5 \pm 8.2 \%$ \\
\hline $\begin{array}{l}\text {...was preferable to using a } \\
\text { pedometer and paper record } \\
(n=37) \text {. }\end{array}$ & $67.6 \pm 7.6 \%$ \\
\hline $\begin{array}{l}\ldots \text { motivated them to be active } \\
\text { and achieve their target } \\
(\mathrm{n}=37) \text {. }\end{array}$ & $81.1 \pm 6.4 \%$ \\
\hline $\begin{array}{l}\ldots \text { motivated them to stay } \\
\text { engaged with the challenge } \\
(n=37) \text {. }\end{array}$ & $83.8 \pm 6.1 \%$ \\
\hline
\end{tabular}

Participants responded positively to the mHealth app design, i.e. the look and feel of the app, see Figure 2. The majority 85.7 ( $\pm 5.4 \%$ ) of participants strongly agreed or agreed that they found the app easy to use and $78.5( \pm 6.3 \%)$ strongly agreed or agreed that they liked the look and feel of the app $(n=37)$. The error used is standard deviation (SD).

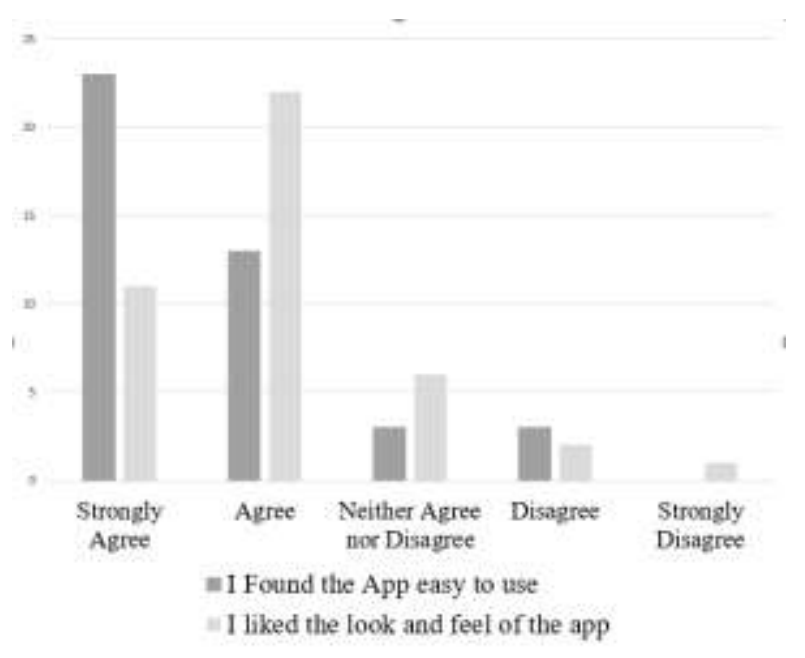

Figure 2. mHealth Application ease of use and interface design $(n=44)$

The mHealth app provided participants with realtime data about their activity levels, which heightened their awareness around activity, and the levels they needed to achieve during the day to reach their activity targets. By using the mHealth tool the availability of the activity data motivated individual users to increase their daily activity. This is highlighted in a number of illustrative quotes from participants. For example, one participant commented "It makes me more aware if I'm not doing any activity and then I try and make up for it" (Team Coordinator1). Another participant noted "The best thing about the app is that you look at it and think oh god I only have three thousand steps. It motivates you to get up and walk around a bit. It gives you an indication of how difficult it actually is to get the ten thousand steps." (Team Coordinator2). Thirty survey participants (where $n=37$ ) indicated that the mHealth platform motivated them to exercise. Team Member3 stated "I do think it can be a great motivator, that in the case of using it you can see a little changes and once you get yourself into a pattern it becomes second nature." However, one participant suggested that they felt the app did not always match the steps tracked by other digital active trackers. But they did not provide examples or evidence to support this.

The leaderboard feature was introduced to the mHealth platform in week 4 of the 5-week "Steps to Health Challenge". This new feature ranked teams according to the number of steps logged by their members. Evidence from the qualitative feedback illustrates that this addition was well received by all challenge participants. Team Member1 commented "You could see what your colleagues are doing and compete a little bit against them". According to Team Coordinator3 "It created a good sense of competition." The increased visibility on team performance enhanced the competition of the challenge, Team Coordinator4 noted "I realised you could see yourself on the leaderboard and that really stepped it up a notch". The perceived increase in competition among the teams was highlighted by another team member. He stated "I found that when I was 500 steps ahead of someone but that gap was closing then it helped me take a lap around the canteen or the office just to get those extra steps to stay ahead" (Team Coordinator3).

The average daily steps taken by an individual during the challenge increased after the leaderboard was introduced. The introduction of this new feature had a positive impact on participants' average number of daily steps. Notably, the average individual daily steps before the leaderboard was 6401 ( \pm 431$)$ steps 
and after was $6690( \pm 489)$ steps $(n=70)$. This average was calculated using the daily steps taken by the 70 active users of the mHealth platform. Due to the sample size, it is not possible to determine whether this increase in steps is statistically significant.

Figure 3 shows that the leaderboard was the most frequently used feature of the mHealth app.

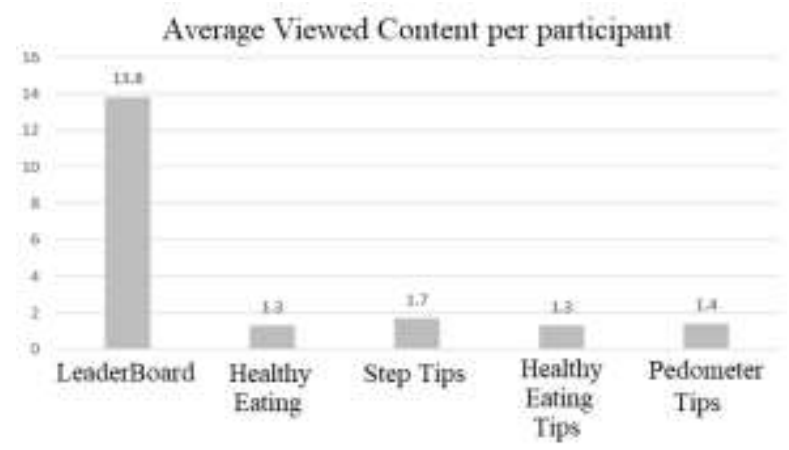

Figure 3. Usage rates across features obtained from the mHealth Application $(n=70)$

For the duration of the study participants indicated a positive or a very positive response to the mHealth app.

Figure 4 shows that most participants felt they would use the digital activity tracking feature again (20 of 37 survey participants).

I would use the app to track my activity again, not as a part of an organized challenge
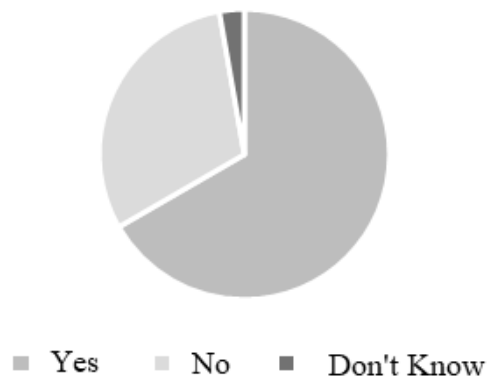

Figures 4. Survey results - using the application in the future for activity tracking $(n=37)$

However, the mood-tracking feature to "track how I feel" did not receive a similar positive response. Figure 5 illustrates that most people indicated they would not use the mood-tracking feature in the future. The negative response to mood-tracking was probed further during the Phase 2 interviews. We received a mixed response, 24 survey participants $(n=37)$ expressed their concern about sharing private and possibly sensitive personal details about how they felt in the workplace.
Two interviewees were concerned about how the data might be used in the future and if their employers should be expected to react if an employee was consistently logging a "sad mood". For example, Team Member2 noted "If a manager gives me a massive amount work or to something I'm not particularly happy about. I wouldn't be comfortable putting it in writing. As there may be a chance that a manager would see it". While Team Coordinator4 commented; "I don't think people would be too forthcoming about putting down their mood if it was their employers looking at it constantly".

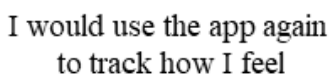
to track how I feel

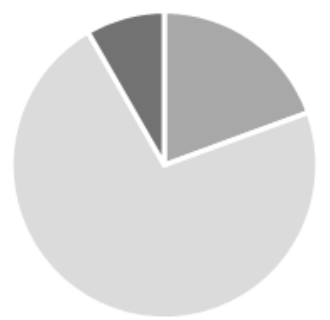

- Yes $=$ No $\square$ Don't Know

Figures 5. Survey results - using the platform in the future for mood-tracking $(n=37)$

Participants also highlighted their concerns about an employers' ability to respond to consistent negative mood logging from an employee. Team Coordinator 1 commented" I would be conscious of somebody who would be reporting low mood all the time, and if there was no action taken. Who's reporting that, who's looking at it. Are they qualified if that person actually needs help?". Another participant questioned the efficacy of the mHealth platform to support someone who needed help, "I am not sure if it is the right intervention for someone who isn't feeling very well, or that it was an appropriate intervention. And I'm not sure about those sad faces, are they a bit frivolous for someone who is feeling very unwell" (Team Coordinator4).

Four of the six interviewees flagged the moodtracking feature as not being particularly useful. For example, one participant commented "I know a lot of people didn't bother putting it in every day or whatever. I'm not sure how truthful they would be or not." (Team Coordinator4). While Team Member1 remarked that "it wasn't something I would find myself using".

It is important to acknowledge that some participants were more positive about the mood tracker. They noted that the capture and subsequent availability of their data over time provided them with 
insights about their mood and how their mood might have impacted their activity in the workplace and vice versa. According to one interviewee "When I was looking back on the few that I put in, it's good to see the changes even in the last couple of weeks. It was useful to look at". Another noted, "That's good you could kind of look back and say, Okay that was probably the reason why I didn't, maybe get to the ten thousand steps" (Team Coordinator3) .

Despite relatively low usage of the mood-tracking feature, it was possible to analyse daily steps in relation to reported mood, see Figure 6.

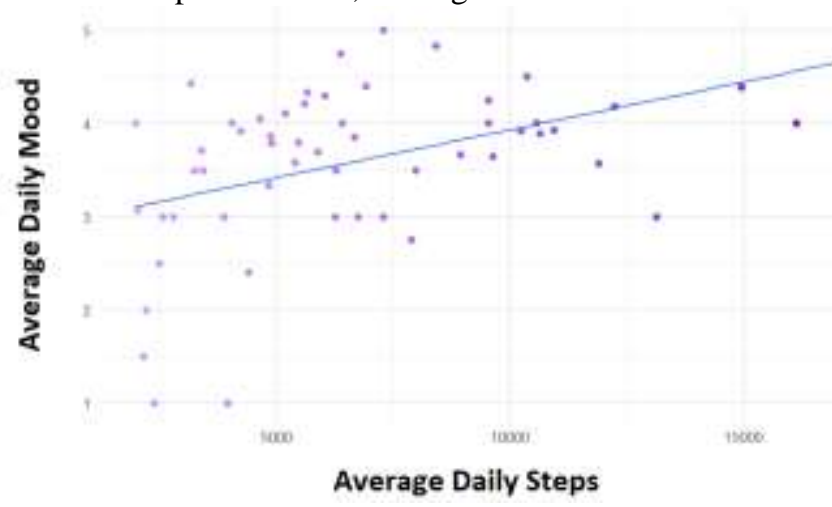

Figure 6. Scatter plot of average daily steps vs average daily mood, with line of best fit included, correlation $r=0.44(n=56)$

A correlation of 0.44 was found between the variables mood and daily activity. This implies that a relationship exists between average daily steps and a participant's mood. However, this does not imply that one causes the other.

Figure 7. illustrates a word cloud of the most commonly used words during the follow-up qualitative interviews. It highlights the words "team" and "good" as the most frequently used by participants. The "team aspect" of the "Steps to Health Challenge" was identified as an important aspect of the WHP initiative.

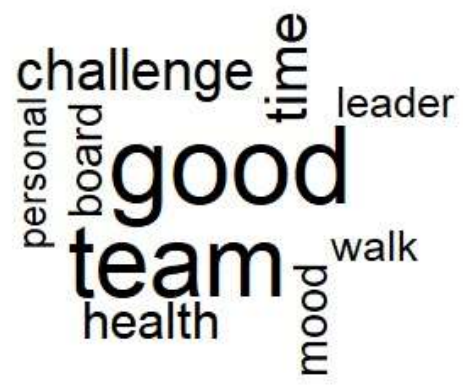

Figure 7. Most frequently used words

All of the interviewees commented that the team aspect of the challenge facilitated positive interactions among staff. Team Coordinator4 stated "I'm relatively new to the HSE so I wouldn't have been that familiar with many people in the building. So that allowed them to get to know me as a new person in the building, I could get to know names. As a result to that, people would come up and talk to you and ask how you're getting on". It seems the "Steps to Health Challenge" provided employees with a common topic for discussion in the workplace, promoting positive engagement, and interaction among individuals, and groups of employees.

\section{Discussion}

Design is important to maintain user engagement with activity-based tracking applications [9, 14]. If an application has a poor user interface it can effect sustained usage of the application and thus effect a user's opinion on the features of the application [28]. The response to the 'look and feel' of the mHealth app was mostly positive. This is supported by the level of engagement with the platform during the five-week challenge.

Half of the interviewees commented that they often forgot to bring their phone when going for a walk. Thus, they felt that the total steps tracked by the application was not an accurate representation of how many steps they had taken each day. Table 1 indicates that some participants felt that the steps logged via the mHealth app did not always match the steps tracked by other digital active trackers used in parallel with the platform. These technologies included other mobile step tracking applications, i.e. a smart watch or Fitbit. However, the survey data showed that approximately half the participants found that it tracked their steps accurately.

Advances in mobile technology makes tracking steps possible [10]. As built-in accelerometers and software used in step tracking applications improve perhaps this issue of accuracy will lessen for future initiatives. However, it is important to consider the effect of human behaviour [10]. The issue of participants forgetting to bring their phone with them while exercising may have negatively affected the accuracy of the step tracking. This problem also exists using a pedometer.

The majority of participants found that using the application made it easier to track their steps and that this intervention was preferable to using a pedometer and paper method. The introduction of an mHealth platform could make it easier for participants to track their steps in future employee health promotion initiatives. The mHealth platform offered a digital alternative to this, which creates new opportunities and challenges for employee WHP initiatives [28]. 
The leaderboard feature elicited positive responses from participants. They commented that this feature introduced a competitive side to the challenge, and it motivated them to increase their step count each day. This mHealth feature provided a form of positive peer pressure, which encouraged employees to increase or maintain their position on the leaderboard by logging a greater number of steps [14]. This was investigated further and a rise in average daily steps was seen after this feature was made available to all participants through the platform [14]. This is an interesting result as one may expect average daily step count to decrease as a challenge continues due to lack of interest or reduction in participation.

Due to the small sample size of app users, it was not possible to ascertain if this rise in step count was statistically significant. The combination of positive results for this feature shows the overall success of the leaderboard as a motivational feature that may be used to positively effect employee behaviour.

The individual mood-tracking feature received mixed responses. The user generated activity dataset indicated that the feature had low user rates. Many participants chose not to use it at all and many used it only occasionally. Three of the six interviewees expressed concerns about individual employee privacy with regard to mood logging, if it were to be included in future WHP initiatives. They were particularly concerned if management would have access to an individual's mood data and how this might be used as employee's mood and attitude toward work is potentially sensitive information. Another recurring challenge identified was around the burden of responsibility of an employer to act upon mood logging data that points to an employee being in a consistently low mood. Some participants commented that it would not be an appropriate mental health intervention for an individual who is experiencing a hard time, calling it "frivolous" and "inappropriate". The mHealth app provided users with the option to log their mood on a scale of 1 to 5. Perhaps a more nuanced approach to mood logging could be designed and evaluated for future versions of the platform [14].

There are a number of limitations to this study. This study did not have access to baseline data of daily steps taken by participants. This data would have provided quantitative evidence to help us better understand if the mHealth app influenced employee behaviour. We conducted a single qualitative case study [29, 30]; we acknowledge that this is a limitation. Multiple case study design increases the rigor of a study through strengthening the exactness, the validity and stability of the findings [31]. A larger sample size, including mHealth platform users, survey respondents, and interviewees, would allow us to more accurately design and assess additional hypotheses. From the data, while not statistically significant it appeared that the distribution of steps taken by men and women were different. Men took, on average, more steps than women but a higher percentage of women had very high daily step counts of greater than 14,000 ( $n=70)$. The study consisted of mostly women, with a sample size of only eight men. In future studies, a larger or more gender balanced sample of participants may provide greater gendered insight into employee behaviour and wellness.

\section{Conclusions}

Digital tools such as activity-based tracking technologies, in the form of smartphones and mobile applications, continue to gain momentum to enable, support, and track employee health promotion programmes [28]. However, they are in their infancy and little is known about their impact on employee behaviour. This study investigates the use of an mHealth activity-based tracking app and its impact on employees' behaviour and wellness as part of an employee health initiative in the HSE.

Using a mixed method approach, this study found that employees were positive about using an mHealth platform to track their daily exercise. We learned more about employees' attitudes to using the platform, employees' reaction to the design of the software application, and specific features and how using the platform may be attributed to changing employee behaviours through the creation of social comparisons [11].

The leaderboard feature was a successful and popular feature among users, promoting positive employee engagement and fun competition as part of this employee WHP initiative. Participants attributed this success to the friendly competitive aspect that it introduced, motivating people to log more steps to improve their own and their team's position on the leaderboard. The leaderboard feature would not have been possible without the introduction of mHealth to the HSE "Steps to Health Challenge". The addition of a digital step counter allowed for steps to be logged and uploaded to the leaderboard in real-time providing a more complete unified and timely view of participants' activities over the duration of the challenge.

The individual mood-tracking feature received mixed responses. The inclusion of this feature allowed for the comparison of participant steps compared to their mood log entries. While some participants enjoyed the feature and found it useful to reflect upon their mood in the past, it seems that further analysis, design, and evaluation is required to fine-tune this 
feature for its inclusion in future initiatives. Additional consideration is required to address concerns around data privacy, the organization's role in accessing this personal mental health-related data, and the action required to support employees in the event of an adverse effect being flagged to the organization.

Clear and precise directions for the use and implementation of mHealth activity-based tracking apps are needed to eliminate uncertainty among employees to secure quality and reach sustainable results [28]. The use of the mHealth app supports users to track and review their personal exercise activity in real-time compared to traditional approaches. In this case, the availability and transparency of individual and team performance positively effected employee activity behaviour. The use of the mHealth platform positively contributed to this initiative and presents a diverse range of opportunities for employee WHP activities in the future. This exploratory study suggests a number of hypotheses for future research, for example:

1. Competition among teams promotes increased employee activity and increased wellness for participants.

2. Control and use of personal data are important to participants in health and wellness programs.

3. Gender influences participation in employee health and wellness programs.

4. Use of trackers with team competition reduces absenteeism and medical costs.

5. Use of trackers with team competition may negatively impact mental health and stress symptoms of some participants.

6. Participant experience using activity tracking technologies positively impacts behavior change and health outcomes.

The first hypothesis about competition among teams is reasonable given our findings and the prior theoretical and empirical literature, c.f. [32, 33]. It remains uncertain if all participants will benefit from the competition. Hypothesis two is based upon the limited use of the mood-tracking feature in this study and general privacy concerns that have been reported about workplace health initiatives, c.f., [34]. The third hypothesis is proposed for future research because of limitations in the sample for this study and because of prior research that showed women are more likely than men to participate in workplace health promotion programs, cf., [35, 36]. Longitudinal research is needed to better understand how workplace health initiatives using trackers and competition can impact absenteeism and medical costs. Also, research is needed about how mHealth interventions, and in particular the use of trackers, can impact mental health and stress symptoms. Finally, future research should examine the impact of participant experience using activity tracking technologies on behavior change and health outcomes.

\section{Acknowledgments}

We would like to thank Innerstength Ltd. for their contribution this study.

\section{References}

[1] World Health Organization (WHO), Health Education and Promotion Unit, "The Health- promoting workplace: making it happen". Geneva. http://www.who.int/iris/handle/10665/64220, 1998.

[2] Martis, L, "7 companies with epic wellness programs", Last accessed 20/9/18 at URL, https://www.monster.com/career-advice/article/companies-good-wellness-programs, 2018.

[3] Aldana, S. G., "Financial impact of health promotion programs: a comprehensive review of the literature", American Journal of Health Promotion, 15(5), 2001, pp. 296320.

[4] Mattila, E., A. L. Orsama, A. Ahtinen, L. Timo Leino, and I. Korhonen, "Personal Health Technologies in Employee Health Promotion: Usage Activity, Usefulness, and Health-Related Outcomes in a 1-Year Randomized Controlled Trial", JMIR mHealth and uHealth, 1(2), 2013.

[5] Emerson, N. D., D. A.Merrill., K. Shedd, R. M. Bilder, and P. Siddarth, "Effects of an employee exercise programme on mental health", Occupational Medicine, 67(2), 2017, pp.128-134.

[6] Stratton, E., A. Lampit, I. Choi , R. A. Calvo, , S. B. Harvey, and N. Glozier, "Effectiveness of eHealth interventions for reducing mental health conditions in employees: A systematic review and meta-analysis", PloS one, 12(12), e0189904, 2017.

[7] Goldberg, L., C. Lockwood, B. Garg, and K. S. Kuehl, "Healthy team healthy $\mathrm{U}$ : a prospective validation of an evidence-based worksite health promotion and wellness platform", Frontiers in public health, 3, 2015, pp.188.

[8] Baker, G., S. R. Gray, A. Wright., C. Fitzsimons, M. Nimmo, R. Lowry, and N. Mutrie, "The effect of a pedometer-based community walking intervention" Walking for Wellbeing in the West" on physical activity levels and health outcomes: a 12-week randomized controlled trial", International Journal of Behavioral Nutrition and Physical Activity, 5(1), 2008, pp. 4.

[9] James, T. L., Deane, J. K., \& Wallace, L. “An application of goal content theory to examine how desired exercise outcomes impact fitness technology feature set selection". Information Systems Journal, 29(5), 2019, pp. 1010-1039. 
[10] Case, M. A., Burwick, H. A., Volpp, K. G., and Patel, M. S., "Accuracy of smartphone applications and wearable devices for tracking physical activity data", JAMA, 313(6), 2015, pp.625-626.

[11] Hamari, J., Hassan, L., \& Dias, A. "Gamification, quantified-self or social networking? Matching users' goals with motivational technology. User Modeling and User Adapted Interaction”, 28(1), 2018, pp.35-74.

[12] Patel, M. S., Asch, D. A., \& Volpp, K. G. "Wearable devices as facilitators, not drivers, of health behavior change". Journal of the American Medical Asso-ciation, 313(5), 2015, pp.459-460.

[13] Y. Wang, I. Weber, P. Mitra "Quantified self meets social media: Sharing of weight updates on Twitter" Proceedings of the 6th International Conference on Digital Health Conference, 2016, pp. 93-97.

[14] Epstein, D. A., Kang, J. H., Pina, L. R., Fogarty, J., \& Munson, S. A. "Reconsidering the device in the drawer: lapses as a design opportunity in personal informatics". In Proceedings of the 2016 ACM International Joint Conference on Pervasive and Ubiquitous Computing, 2016, pp. 829-840.

[15] Harrison, D., Marshall, P., Bianchi-Berthouze, N., \& Bird, J. "Activity tracking: barriers, workarounds and customisation". In Proceedings of the 2015 ACM International Joint Conference on Pervasive and Ubiquitous Computing, 2015, pp. 617-621.

[16] Lowry, P. B., Gaskin, J., \& Moody, G. D. "Proposing the multi-motive information systems continuance model (MISC) to better explain end-user system evaluations and continuance intentions", Journal of the Association for Information Systems, 16(7), 2015, pp.515-579.

[17] Deci, E. L. \& Ryan, R. M. (1995). "Human autonomy: The basis for true self-esteem". In M. Kemis (Ed.), Efficacy, Agency, and Self-esteem (pp. 31-49). New York, NY, USA: Plenum.

[18] Yang, Y., Yang, Y., \& Teo, H. H. "Optimal design of gamified incentive hierarchies in online fitness communities". Paper presented at the 38th International Conference on Information Systems, Seoul, South Korea, 2017.

[19] Consolvo, S., Everitt, K., Smith, I., \& Landay, J. A. "Design requirements for technologies that encourage physical activity". In Proceedings of the SIGCHI conference on human factors in computing systems, NewYork, NY, USA: ACM, 2006, pp. 457-466.

[20] Chan, C. B., D. A Ryan, and C. Tudor-Locke, "Health benefits of a pedometer-based physical activity intervention in sedentary workers", Preventive medicine, 39(6), 2004, pp.1215-1222.

[21] Street, T. D., and S. J. Lacey, "Employee Perceptions of Workplace Health Promotion Programs: Comparison of a
Tailored, Semi-Tailored, and Standardized Approach. International journal of environmental research and public health", 15(5), 2018.

[22] Miyamoto, S. W., S. Henderson, H.M. Young, A. Pande, and J. J. Han, "Tracking health data is not enough: a qualitative exploration of the role of healthcare partnerships and mHealth technology to promote physical activity and to sustain behavior change", JMIR mHealth and uHealth, 4(1), 2016.

[23] Gilson, N. D., G. Faulkner, M.H. Murphy, M.R.U. Meyer, T. Washington, , G.C. Ryde, K.A. Dillon, "Walk@ Work: An automated intervention to increase walking in university employees not achieving 10,000 daily steps", Preventive medicine, 56(5), 2013, pp.283-287.

[24] Price, M., E. K. Yuen, E. M. Goetter, J. D. Herbert, E. M. Forman, R. Acierno, and K. J. Ruggiero, "mHealth: a mechanism to deliver more accessible, more effective mental health care", Clinical psychology \& psychotherapy, 21(5), 2014, pp.427-436

[25] Creswell, J.W., Fetters, M.D. and Ivankova, N.V. "Designing a mixed methods study in primary care". The Annals of Family Medicine, 2, 2004, pp.7-12.

[26] Brooke, J. "SUS-A quick and dirty usability scale". Usability evaluation in industry, 189(194), 1996, pp.4-7.

[27] Strauss, A. and Corbin, J. "Basics of Qualitative Research: Techniques and Procedures for Developing Grounded Theory”, Sage Publications, California, 1990.

[28] Jimenez, P. and A. Bregenzer, "Integration of eHealth Tools in the Process of Workplace Health Promotion: Proposal for Design and Implementation", Journal of medical Internet research, 20(2), 2018.

[29] Cavaye, A. L. M., “Case study research: A multi-faceted research approach for IS", Information Systems Journal, 6, 1996, pp. 227-242.

[30] Darke, P., G. Shanks, and M. Broadbent, "Successfully Completing Case Study Research: Combining Rigour, Relevance and Pragmatism", Information Systems Journal, 8(4), 1998, pp. 273-289.

[31] Yin, R., Case study research: "Design and methods. Applied Social Research Methods Series", Sage Publications, London, 2009.

[32] Deutsch, M., "A theory of co-operation and competition," Human Relations, 1949, pp. 129-152.

[33] Marker, A. M. and A. E. Staiano, "Better Together: Outcomes of Cooperation versus Competition in Social Exergaming," Games For Health Journal, 4(1), 2015, pp. 2530. 